\section{Predictive Value of Musculoskeletal Ultrasound for Flares in Juvenile Idiopathic Arthritis}

\section{To the Editor:}

I have read with great interest the recent publication by Zhao, et al $^{1}$ on the predictive value of musculoskeletal ultrasound for flares of patients with juvenile idiopathic arthritis that is clinically inactive. Based on their evaluation, the authors suggest that the positive predictive value (PPV) of ultrasound (US) findings for clinical flares is very low at $12 \%$ and that a second US had even less PPV. The authors describe that synovitis on US was assessed through B-mode, identifying effusion and synovial hypertrophy as well as Doppler US

Looking at their results and one table in particular, it is nevertheless striking that 11 wrist joints were considered abnormal on US with all of them being Doppler-positive, but only 1 had abnormal findings on B-mode. This is even more significant for the tibiotalar joint.

From a histologic as well as an US point of view, synovitis is defined as synovial proliferation with increased synovial fluid secretion and associated hyperemia of existing and newly formed blood vessels. The detection of blood flow without structural changes of the synovium (detectable on B-mode) cannot qualify as synovitis. Both the adult US synovitis definition the authors used ${ }^{2}$ as well as more recent pediatric definitions ${ }^{3}$ are very clear on this. Doppler signals have to be detected within an area of abnormal synovium. Blood flow can be detected within a joint but outside the synovium (because many joints have intraarticular tissue that is not synovium), but these Doppler signals cannot qualify as synovitis ${ }^{4}$.

For the wrist, only 1 wrist would therefore qualify as abnormal on US and none of the tibiotalar joints. The authors might be describing physiologic blood flow as abnormal US findings. In the discussion section, the authors partially acknowledge this problem, suggesting that they may have located increased blood flow as part of synovitis without visible synovial changes on B-mode. This statement is not supported by the literature and is difficult to understand given the excellent spatial resolution of US. The conclusion that nearly half of the children had abnormal US findings is not supported by the data.

Many clinicians doing US have observed increased blood flow surrounding distended synovium and the apparent persistence of intraarticular or periarticular hyperemia in joints even with the resolution of structural synovial changes on B-mode. To my knowledge, there are no data available to date that would provide methodological guidance on how to distinguish such presumed abnormal blood flow from physiologic intraarticular blood flow in the absence of abnormal structural findings of the synovium.

The assessment of some joints is also incomplete in this publication ${ }^{1}$. For example, the subtalar joint was assessed through 1 view from lateral only. The subtalar joint is nevertheless a complex joint in itself that has an anterior part and a posterior part, and the assessment needs to be done through several views to record pathology reliably ${ }^{5}$. Pediatric-specific publications demonstrating this and the need to assess the subtalar joint from medial and lateral have been available for several years ${ }^{6}$ and are even cited by the authors.

The US data presented in the manuscript do not support the conclusions drawn by the authors. Interestingly, another recent publication came to a different conclusion on the predictive value of US examinations for clinical flares, although it did rely on 1 assessment at baseline only ${ }^{7}$.

JOHANNES ROTH, MD, PhD, FRCPC, RhMSUS, Children's Hospital of Eastern Ontario, 401 Smyth Road, Ottawa, Ontario K1H 8L1, Canada. Address correspondence to Dr. J. Roth. E-mail: jroth@ cheo.on.ca

\section{REFERENCES}

1. Zhao Y, Rascoff NE, Iyer RS, Thapa M, Reichley L, Oron AP, et al. Flares of disease in children with clinically inactive juvenile idiopathic arthritis were not correlated with ultrasound findings. J Rheumatol 2018;45:851-7.

2. Wakefield RJ, Balint PV, Szkudlarek M, Filippucci E, Backhaus M, D'Agostino MA, et al. OMERACT 7 Special Interest Group. Musculoskeletal ultrasound including definitions for ultrasonographic pathology. J Rheumatol 2005;32:2485-7

3. Roth J, Ravagnani V, Backhaus M, Balint P, Bruns A, Bruyn GA, et al. Preliminary definitions for the sonographic features of synovitis in children. Arthritis Care Res 2017;69:1217-23

4. Collado P, Windschall D, Vojinovic J, Magni-Manzoni S, Balint P, Bruyn GAW, et al. Amendment of the OMERACT ultrasound definitions of joints' features in healthy children when using the DOPPLER technique. Pediatr Rheumatol Online J 2018;16:23.

5. Mandl P, Bong D, Balint PV, Hammer HB, Miguel M, Naredo E, et al. Sonographic and anatomic description of the subtalar joint. Ultrasound Med Biol 2018;44:119-23.

6. Laurell L, Court-Payen M, Nielsen S, Zak M, Fasth A Ultrasonography and color Doppler in juvenile idiopathic arthritis: diagnosis and follow-up of ultrasound-guided steroid injection in the ankle region. A descriptive interventional study. Pediatr Rheumatol Online J 2011;9:4.

7. De Lucia O, Ravagnani V, Pregnolato F, Hila A, Pontikaki I, Gattinara M, et al. Baseline ultrasound examination as possible predictor of relapse in patients affected by juvenile idiopathic arthritis (JIA). Ann Rheum Dis 2018 Feb 6 (E-pub ahead of print).

J Rheumatol 2019;46:1; doi:10.3899/jrheum.180735 